\title{
Changes in electrocardiographic findings after closed thoracostomy in patients with spontaneous pneumothorax
}

\author{
Wonjae Lee ${ }^{1}$, Yoonje Lee ${ }^{1}$, Changsun Kim ${ }^{1}$, Hyuk Joong Choi ${ }^{1}$, \\ Bossng Kang ${ }^{1}$, Tae Ho Lim², Jaehoon $\mathrm{Oh}^{2}{ }^{2}$, Hyunggoo Kang ${ }^{2}$, \\ Junghun Shin ${ }^{3}$
}

\begin{abstract}
'Department of Emergency Medicine, Hanyang University Guri Hospital, Guri, Korea ${ }^{2}$ Department of Emergency Medicine, Hanyang University Seoul Hospital, Seoul, Korea ${ }^{3}$ Department of Cardiology, Hanyang University Guri Hospital, Guri, Korea
\end{abstract}

Objective We aimed to describe electrocardiographic (ECG) findings in spontaneous pneumothorax patients before and after closed thoracostomy.

Methods This is a retrospective study which included patients with spontaneous pneumothorax who presented to an emergency department of a tertiary urban hospital from February 2005 to March 2015. The primary outcome was a difference in ECG findings between before and after closed thoracostomy. We specifically investigated the following ECG elements: PR, QRS, OTC, axis, ST segments, and R waves in each lead. The secondary outcomes were change in ST segment in any lead and change in axis after closed thoracostomy.

Results There were two ECG elements which showed statistically significant difference after thoracostomy. With right pneumothorax volume of greater than $80 \%$, QTc and the R waves in aVF and V5 significantly changed after thoracostomy. With left pneumothorax volume between $31 \%$ and $80 \%$, the ST segment in V2 and the R wave in V1 significantly changed after thoracostomy. However, majority of ECG elements did not show statistically significant alteration after thoracostomy.

Conclusion We found only minor changes in ECG after closed thoracostomy in spontaneous pneumothorax patients.

Keywords Electrocardiography; Pneumothorax; Tension pneumothorax
elSSN: 2383-4625

Received: 8 October 2016 Revised: 26 November 2016

Accepted: 2 December 2016

Correspondence to: Yoonje Lee Department of Emergency Medicine, Hanyang University Guri Hospital, 153 Gyeongchun-ro, Guri 11923, Korea E-mail:yong0831@naver.com

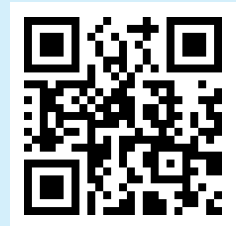

How to cite this article:

Lee W, Lee Y, Kim C, Choi HJ, Kang B, Lim $\mathrm{TH}, \mathrm{Oh} J$, Kang $\mathrm{H}$, Shin J. Changes in electrocardiographic findings in patients with spontaneous pneumothorax. Clin Exp Emerg Med 2017;4(1):38-47.

This is an Open Access article distributed under the terms of the Creative Commons Attribution Non-Commercial License (http:// creativecommons.org/licenses/by-nc/4.0/). 


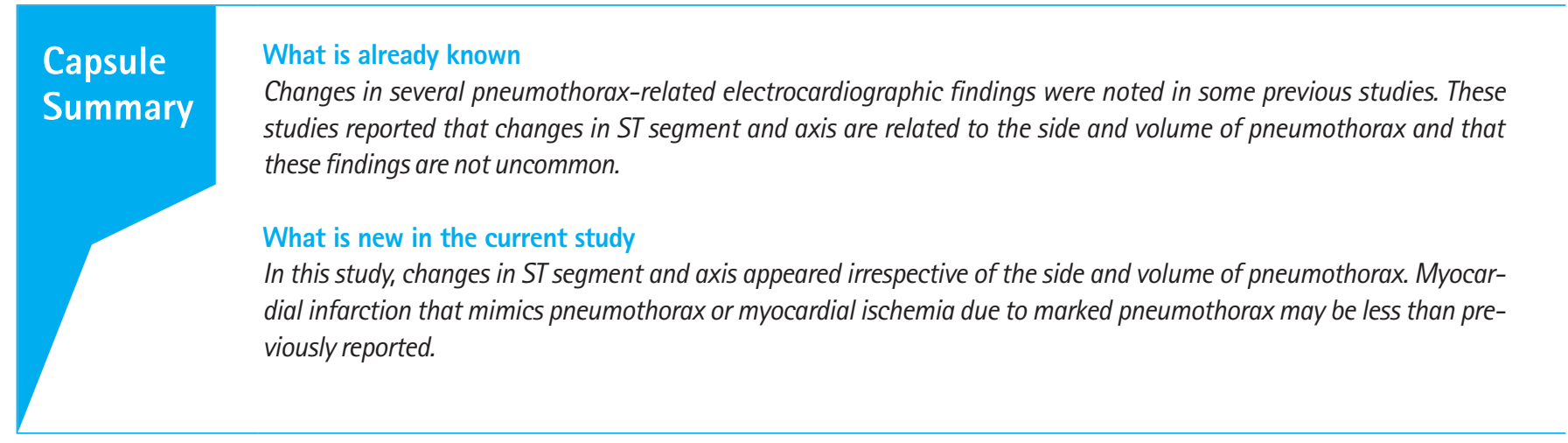

\section{INTRODUCTION}

Many diseases mimic myocardial infarction. Most physicians consider an acute coronary syndrome in patients with chest pain, dyspnea, and electrocardiographic (ECG) changes consisting of ST segment deviation or interventricular conduction delay findings, among other features. Interest in ECG changes in patients with pneumothorax began to develop in the first half of the 20th century, and many pulmonary tuberculosis patients managed with artificial pneumothorax were a common research focus. ${ }^{1}$ A few years later, researchers were concerned owing to the overlapping patterns of ECG changes in between patients with pneumothorax and those with other serious diseases such as pulmonary embolism and myocardial infarction. ${ }^{2-4}$

As observed in these studies, certain patients with symptoms of suspected acute coronary syndrome are diagnosed with other diseases such as pneumothorax, pneumonia, costochondritis, or a myofascial pain syndrome of the intercostal muscles. ${ }^{2-4}$ Among these diseases, pneumothorax is a condition that may mimic myocardial infarction. Although myocardial infarction is a more serious medical emergency, pneumothorax may be a lethal disease. In a previous study, researchers investigated ECG changes in ST segments in patients with pneumothorax; however, the results were not sufficient to explain the ECG changes. ${ }^{5}$ In another study, researchers evaluated the prevalence of ECG changes in patients with spontaneous pneumothorax, assessed the relationship between ECG changes and the size and side of pneumothorax, and analyzed the degree of hypoxemia as the potential underlying mechanism of the ECG changes in patients with pneumothorax. ${ }^{6}$ The researchers reported that ECG findings in patients with pneumothorax often reveal significant abnormalities. ${ }^{6}$ However, these studies were conducted with a small sample size. ${ }^{5,6}$ In addition, they reported that the most common ECG changes in pneumothorax include right axis deviation, reduced QRS amplitude in precordial leads, and T-wave inversion. In most cases, the ECG changes revert to normal after resolution of the pneumo- thorax and are not related to any underlying cardiac diseases. ${ }^{78}$ To the best of our knowledge, ECG changes in patients with pneumothorax after closed thoracostomy has not been investigated to date.

Therefore, we hypothesized that specific ECG changes in patients with pneumothorax could depend on the side and/or volume of the pneumothorax, consistent with the results of previous studies, but also that this is not a general principle, in accordance with certain results from other studies that contradict those of previous studies in regard to ST segments, $R$ wave voltage, QRS axis, among others. ${ }^{5-8}$ In this study, we specifically aimed to evaluate the prevalence of ECG changes in patients with pneumothorax, and assess the relationship between ECG changes (ST segments, PR interval, QRS interval, QTc interval, axis, and R wave amplitude) and the size and side of pneumothorax, and compare ECG findings before and after closed thoracostomy.

\section{METHODS}

\section{Study design and setting}

A retrospective study was conducted by using data prospectively collected for 11 years. The study was performed in the academic emergency department of a tertiary urban hospital from February 2004 to March 2015 and was approved and documentation of consent has been waived by our institutional review board.

\section{Study participants}

Patients with pneumothorax who were registered in the emergency department between February 2004 and March 2015 were included in this study. Among these patients, those with ECG findings from only before or after closed thoracostomy and those with trauma-related pneumothorax or underlying cardiac/pulmonary disease (restrictive lung disease with or without pleural free fluids) were excluded.

In total, 389 patients with spontaneous pneumothorax were enrolled. Finally, the data of 344 patients were excluded because 
the patients underwent electrocardiography either only before or only after closed thoracostomy. Therefore, the data of 45 patients were included in this study.

In this study, we divided the enrolled patients' data into the following 3 groups based on the volume of the pneumothorax: $<30 \%$, between $31 \%$ and $80 \%$, and $>80 \%$. This grouping had not been performed in any previous studies. However, many previous studies reported that the volume of the pneumothorax might be an influencing factor of abnormal ECG findings. Thus, we applied this grouping in the present study.

\section{Outcomes}

The baseline characteristics of the patients included sex, age, and the side and volume of pneumothorax. The pneumothorax volume was measured by using the Collins method.

The primary outcome was the change in ECG findings after closed thoracostomy. In this study, we specifically investigated the following ECG findings: PR, QRS, corrected OT interval, axis, ST segments, and $R$ waves in each lead. The PR interval, QRS axis, and QTc interval were measured by using a 12-lead ECG machine and the ST segments and $\mathrm{R}$ waves were measured using a caliper. In particular, the ST segments were measured from the J point.

The secondary outcomes were analyzed with cases of change in ST segments in any lead after closed thoracostomy and change in axis after closed thoracostomy.

\section{Statistical analysis}

Data were compiled by using a standard spreadsheet application (Excel; Microsoft, Redmond, WA, USA) and analyzed by using the IBM SPSS ver. 20.0 (IBM Corp., Armonk, NY, USA). We generated descriptive statistics and present categorical data as frequencies and percentages or continuous data as means with the standard deviation.

To evaluate the differences in ECG findings after closed thoracostomy, the paired t-test was used for data with a normal distribution and the Wilcoxon signed-rank test was used for data with a non-normal distribution. For all the analyzed data, a P-value $<0.05$ was considered statistically significant.

\section{RESULTS}

\section{Baseline characteristics}

The baseline characteristics of the enrolled patients are shown in Table 1. Of the cases, 25 were left-sided pneumothorax and 20 were right-sided pneumothorax. According to the Collins method, the mean volume of the right-sided pneumothorax was $60.2 \pm$ $30.1 \%$, and that of the left-sided pneumothorax was $50.5 \pm 27.0 \%$.
Table 1. Baseline characteristics of the patients

\begin{tabular}{lcc}
\hline Characteristics & Right side $(\mathrm{n}=20)$ & Left side $(\mathrm{n}=25)$ \\
\hline Sex, male & $16(80)$ & $22(88)$ \\
Age (yr) & $31.9 \pm 21.1$ & $40.9 \pm 24.8$ \\
$\begin{array}{l}\text { Pneumothorax volume (\%) } \\
\text { No. of cases in each range of }\end{array}$ & $60.2 \pm 30.1$ & $50.5 \pm 27.0$ \\
$\begin{array}{l}\text { pneumothorax volume } \\
<30 \%\end{array}$ & $3(15)$ & \\
$30 \%-80 \%$ & $9(45)$ & $5(20)$ \\
$>80 \%$ & $8(40)$ & $5(20)$ \\
\hline
\end{tabular}

Values are presented as number (\%) or mean \pm standard deviation.

We also describe the numbers of cases according to the volume of the pneumothorax in Table 1.

\section{Comparison of various ECG findings before and after closed thoracostomy according to the volume of right- sided pneumothorax}

Most of the changes in ECG findings in right-sided pneumothorax after closed thoracostomy were not statistically significant. Only in cases of a pneumothorax volume of $>80 \%$ were QTc and the $R$ waves in leads aVF and V 5 significantly different after closed thoracostomy (Table 2).

\section{Comparison of various ECG findings before and after closed thoracostomy according to the volume of left- sided pneumothorax}

Most of the changes in ECG findings in left-sided pneumothorax after closed thoracostomy were not statistically significant. Only in cases of a pneumothorax volume from 30\% to 80\% were the ST segment in lead V2 and the R wave in lead V1 significantly different after closed thoracostomy (Table 3).

\section{Changes in ST segment after closed thoracostomy}

In the right-sided pneumothorax cases, a ST segment abnormality was observed in 11 cases $(11 / 20,55 \%)$, of which only $3(3 / 11$, 27\%) recovered a normal ST segment. In the left-sided pneumothorax cases, a ST segment abnormality was observed in 8 cases $(8 / 25,32 \%)$, of which only $2(2 / 8,25 \%)$ recovered a normal ST segment (Table 4).

Among the right-sided pneumothorax cases, the numbers of cases with ST segment abnormality according to pneumothorax volumes of $<30 \%$, between $31 \%$ and $80 \%$, and of $>80 \%$ were 2,4 , and 5, respectively. Among the left-sided pneumothorax cases, the numbers of cases with ST segment abnormality and pneumothorax volumes of $<30 \%$, between $31 \%$ and $80 \%$, and of $>80 \%$ were 2,5 , and 1 , respectively. 
Table 2. Comparison of various ECG findings before and after closed thoracostomy according to the volume of right-sided pneumothorax

\begin{tabular}{|c|c|c|c|c|c|c|c|c|}
\hline \multicolumn{3}{|c|}{ Volume $<30 \%$} & \multicolumn{3}{|c|}{ Volume $30 \%-80 \%$} & \multicolumn{3}{|c|}{ Volume $>80 \%$} \\
\hline \multicolumn{2}{|c|}{ ECG values } & \multirow{2}{*}{$\begin{array}{c}\text { P-value } \\
0.29\end{array}$} & \multicolumn{2}{|c|}{ ECG values } & \multirow{2}{*}{$\begin{array}{c}\text { P-value } \\
0.86\end{array}$} & \multicolumn{2}{|c|}{ ECG values } & \multirow{2}{*}{$\begin{array}{r}\text { P-value } \\
0.67\end{array}$} \\
\hline Pre-axis & $66 \pm 0$ & & Pre-axis & $74 \pm 29$ & & Pre-axis & $89.5 \pm 34$ & \\
\hline Post-axis & $72 \pm 0$ & & Post-axis & $70 \pm 37$ & & Post-axis & $76.5 \pm 30$ & \\
\hline Pre-PR & $140 \pm 0$ & 0.28 & Pre-PR & $154 \pm 48$ & 0.78 & Pre-PR & $148.5 \pm 15$ & 0.87 \\
\hline Post-PR & $141.7 \pm 11.6$ & & Post-PR & $162.8 \pm 33.4$ & & Post-PR & $148.8 \pm 21.9$ & \\
\hline Pre-QRS & $92.7 \pm 17.2$ & 0.41 & Pre-QRS & $93.3 \pm 7.1$ & 0.23 & Pre-QRS & $99.8 \pm 9.5$ & 0.61 \\
\hline Post-QRS & $102 \pm 0$ & & Post-QRS & $90 \pm 12$ & & Post-QRS & $106 \pm 23$ & \\
\hline Pre-OTc & $404.7 \pm 28.2$ & 0.96 & Pre-QTc & $406 \pm 25.4$ & 0.91 & Pre-QTc & $427.1 \pm 14.4$ & $0.01^{*}$ \\
\hline Post-OTc & $405.3 \pm 24.9$ & & Post-0Tc & $407.3 \pm 11.9$ & & Post-OTc & $406.9 \pm 19.8$ & \\
\hline Pre-I ST & 0 & 1.0 & Pre-I ST & 0 & 1.0 & Pre-I ST & $0.1 \pm 0.4$ & 0.32 \\
\hline Post-I ST & 0 & & Post-I ST & 0 & & Post-I ST & 0 & \\
\hline Pre-II ST & 0 & 1.0 & Pre-II ST & 0 & 1.0 & Pre-II ST & $0.1 \pm 0.4$ & 0.32 \\
\hline Post-II ST & 0 & & Post-II ST & 0 & & Post-II ST & 0 & \\
\hline Pre-III ST & 0 & 1.0 & Pre-III ST & 0 & 1.0 & Pre-III ST & $0.1 \pm 0.4$ & 0.32 \\
\hline Post-III ST & 0 & & Post-III ST & 0 & & Post-III ST & 0 & \\
\hline Pre-aVR ST & 0 & 1.0 & Pre-aVR ST & 0 & 1.0 & Pre-aVR ST & 0 & 1.0 \\
\hline Post-aVR ST & 0 & & Post-aVR ST & 0 & & Post-aVR ST & 0 & \\
\hline Pre-aVL ST & 0 & 1.0 & Pre-aVL ST & 0 & 1.0 & Pre-aVL ST & $0.1 \pm 0.4$ & 0.32 \\
\hline Post-aVL ST & 0 & & Post-aVL ST & 0 & & Post-aVL ST & 0 & \\
\hline Pre-aVF ST & 0 & 1.0 & Pre-aVF ST & 0 & 1.0 & Pre-aVF ST & $0.1 \pm 0.4$ & 0.32 \\
\hline Post-aVF ST & 0 & & Post-aVF ST & 0 & & Post-aVF ST & 0 & \\
\hline Pre-V1 ST & 0 & 0.32 & Pre-V1 ST & $0.1 \pm 0.3$ & 0.32 & Pre-V1 ST & 0 & 0.32 \\
\hline Post-V1 ST & $0.3 \pm 0.6$ & & Post-V1 ST & 0 & & Post-V1 ST & $0.1 \pm 0.4$ & \\
\hline Pre-V2 ST & 0 & 0.32 & Pre-V2 ST & $0.4 \pm 0.6$ & 0.41 & Pre-V2 ST & $0.4 \pm 0.7$ & 0.28 \\
\hline Post-V2 ST & $0.3 \pm 0.6$ & & Post-V2 ST & $0.2 \pm 0.7$ & & Post-V2 ST & $0.6 \pm 0.6$ & \\
\hline Pre-V3 ST & $0.3 \pm 0.6$ & 0.32 & Pre-V3 ST & $0.7 \pm 0.9$ & 0.58 & Pre-V3 ST & $0.8 \pm 0.8$ & 0.79 \\
\hline Post-V3 ST & $0.7 \pm 1.2$ & & Post-V3 ST & $0.6 \pm 0.8$ & & Post-V3 ST & $0.7 \pm 0.8$ & \\
\hline Pre-V4 ST & $0.5 \pm 0.9$ & 0.32 & Pre-V4 ST & $0.4 \pm 0.6$ & 0.85 & Pre-V4 ST & $0.5 \pm 0.8$ & 0.41 \\
\hline Post-V4 ST & $0.3 \pm 0.6$ & & Post-V4 ST & $0.4 \pm 0.7$ & & Post-V4 ST & $0.3 \pm 0.5$ & \\
\hline Pre-V5 ST & $0.8 \pm 0.8$ & 0.66 & Pre-V5 ST & $0.1 \pm 0.6$ & 0.89 & Pre-V5 ST & $0.4 \pm 0.5$ & 0.32 \\
\hline Post-V5 ST & $0.7 \pm 1.2$ & & Post-V5 ST & $0.1 \pm 0.3$ & & Post-V5 ST & $0.3 \pm 0.5$ & \\
\hline Pre-V6 ST & 0 & 0.32 & Pre-V6 ST & 0 & 1.0 & Pre-V6 ST & $0.3 \pm 0.5$ & 0.32 \\
\hline Post-V6 ST & $0.3 \pm 0.6$ & & Post-V6 ST & 0 & & Post-V6 ST & $0.1 \pm 0.4$ & \\
\hline Pre-I R & $5 \pm 0$ & 0.1 & Pre-I R & $4 \pm 2$ & 0.67 & Pre-I R & $1 \pm 3$ & 0.17 \\
\hline Post-I R & $4 \pm 1$ & & Post-I R & $3.6 \pm 2$ & & Post-I R & $2.9 \pm 1.6$ & \\
\hline Pre-II R & $10.3 \pm 2.5$ & 0.42 & Pre-II R & $9.8 \pm 4.5$ & 0.91 & Pre-II R & $11 \pm 4.8$ & 0.08 \\
\hline Post-II R & $11 \pm 3.6$ & & Post-II R & $9.7 \pm 3.5$ & & Post-II R & $9.8 \pm 3.9$ & \\
\hline Pre-III R & $5.3 \pm 4.2$ & 0.19 & Pre-III R & $8.3 \pm 4.1$ & 0.43 & Pre-III R & $10.5 \pm 6.9$ & 0.29 \\
\hline Post-III R & $7.0 \pm 2.6$ & & Post-III R & $7.3 \pm 3.5$ & & Post-III R & $9.0 \pm 5.8$ & \\
\hline Pre-aVR R & $1 \pm 0$ & 0.32 & Pre-aVR R & $1 \pm 1$ & 0.74 & Pre-aVR R & $1.8 \pm 4$ & 0.45 \\
\hline Post-aVR R & 0 & & Post-aVR R & $1 \pm 2$ & & Post-aVR R & $1.5 \pm 4$ & \\
\hline Pre-aVL R & $3 \pm 0$ & 0.16 & Pre-aVL R & $2 \pm 2$ & 0.32 & Pre-aVL R & $1.5 \pm 1$ & 0.71 \\
\hline Post-aVL R & $2 \pm 0$ & & Post-aVL R & $1 \pm 2$ & & Post-aVL R & $1 \pm 2$ & \\
\hline Pre-aVF R & $8.3 \pm 3.2$ & 0.16 & Pre-aVF R & $9 \pm 3.9$ & 0.33 & Pre-aVF R & $10.8 \pm 5.8$ & 0.05 \\
\hline Post-aVF R & $7 \pm 0$ & & Post-aVF R & $8 \pm 5$ & & Post-aVF R & $7.5 \pm 10$ & \\
\hline Pre-V1 R & 0 & 0.16 & Pre-V1 R & $2 \pm 5$ & 0.49 & Pre-V1 R & $2 \pm 4$ & 0.49 \\
\hline Post-V1 R & $3 \pm 0$ & & Post-V1 R & $2 \pm 3$ & & Post-V1 R & $3.5 \pm 3$ & \\
\hline Pre-V2 R & $3.3 \pm 2.1$ & 0.07 & Pre-V2 R & $7.3 \pm 5.7$ & 0.17 & Pre-V2 R & $5.9 \pm 3.2$ & 0.81 \\
\hline Post-V2 R & $5.7 \pm 2.1$ & & Post-V2 R & $5.7 \pm 4$ & & Post-V2 R & $6.1 \pm 2.9$ & \\
\hline Pre-V3 R & $6 \pm 2.6$ & 0.32 & Pre-V3 R & $8.9 \pm 6.5$ & 0.82 & Pre-V3 R & $9.6 \pm 6.2$ & 0.19 \\
\hline Post-V3 R & $8 \pm 4.6$ & & Post-V3 R & $8.6 \pm 4.9$ & & Post-V3 R & $7.1 \pm 3.7$ & \\
\hline Pre-V4 R & $15.7 \pm 3.1$ & 0.37 & Pre-V4 R & $13 \pm 7.9$ & 0.24 & Pre-V4 R & $13.4 \pm 5.2$ & 0.49 \\
\hline Post-V4 R & $13 \pm 0$ & & Post-V4 R & $15 \pm 4$ & & Post-V4 R & $10.5 \pm 8$ & \\
\hline Pre-V5 R & $18 \pm 0$ & 0.18 & Pre-V5 R & $15 \pm 7$ & 0.67 & Pre-V5 R & $14.5 \pm 11$ & $0.03^{*}$ \\
\hline Post-V5 R & $22 \pm 4.6$ & & Post-V5 R & $13.7 \pm 3$ & & Post-V5 R & $11.5 \pm 6.3$ & \\
\hline Pre-V6 R & $11 \pm 0$ & 0.11 & Pre-V6 R & $12 \pm 9$ & 0.53 & Pre-V6 R & $13 \pm 8$ & 0.12 \\
\hline Post-V6 R & $15 \pm 0$ & & Post-V6 R & $11 \pm 4$ & & Post-V6 R & $8.5 \pm 7$ & \\
\hline
\end{tabular}

Continuous variables are presented as mean \pm standard deviation.

ECG, electrocardiographic; Pre, before closed thoracostomy state; Post, after closed thoracostomy state.

${ }^{*} \mathrm{P}<0.05$ is statistically significant. 
Table 3. Comparison of various ECG findings before and after closed thoracostomy according to the volume of left-sided pneumothorax

\begin{tabular}{|c|c|c|c|c|c|c|c|c|}
\hline \multicolumn{3}{|c|}{ Volume $<30 \%$} & \multicolumn{3}{|c|}{ Volume $30 \%-80 \%$} & \multicolumn{3}{|c|}{ Volume $>80 \%$} \\
\hline \multicolumn{2}{|c|}{ ECG values } & \multirow{2}{*}{$\begin{array}{c}\text { P-value } \\
0.69\end{array}$} & \multicolumn{2}{|c|}{ ECG values } & \multirow{2}{*}{$\begin{array}{c}\text { P-value } \\
0.17\end{array}$} & \multicolumn{2}{|c|}{ ECG values } & \multirow{2}{*}{$\begin{array}{r}\text { P-value } \\
0.89\end{array}$} \\
\hline Pre-axis & $85 \pm 50$ & & Pre-axis & $67 \pm 41$ & & Pre-axis & $87 \pm 108$ & \\
\hline Post-axis & $84 \pm 8$ & & Post-axis & $52 \pm 41$ & & Post-axis & $59 \pm 68$ & \\
\hline Pre-PR & $146.8 \pm 23.3$ & 0.5 & Pre-PR & $159.2 \pm 17.4$ & 0.32 & Pre-PR & $148.4 \pm 15.4$ & 0.96 \\
\hline Post-PR & $174.2 \pm 60.5$ & & Post-PR & $157.9 \pm 15.2$ & & Post-PR & $148 \pm 11.6$ & \\
\hline Pre-QRS & $96 \pm 7.1$ & 0.43 & Pre-ORS & $89.5 \pm 10.3$ & 0.09 & Pre-QRS & $90.8 \pm 7.2$ & 0.49 \\
\hline Post-QRS & $90.4 \pm 13.1$ & & Post-QRS & $93.2 \pm 8.1$ & & Post-QRS & $94.4 \pm 11.8$ & \\
\hline Pre-0Tc & $405 \pm 20$ & 0.22 & Pre-0Tc & $402.0 \pm 52$ & 0.97 & Pre-OTc & $398 \pm 16$ & 0.14 \\
\hline Post-QTc & $422.6 \pm 28.7$ & & Post-0Tc & $409.9 \pm 24.4$ & & Post-QTc & $412.4 \pm 16.6$ & \\
\hline Pre-I ST & $0.2 \pm 0.4$ & 1.0 & Pre-I ST & 0 & 0.32 & Pre-I ST & 0 & 1.0 \\
\hline Post-I ST & $0.2 \pm 0.4$ & & Post-I ST & $0.1 \pm 0.3$ & & Post-I ST & 0 & \\
\hline Pre-II ST & $0.2 \pm 0.4$ & 0.32 & Pre-II ST & $-0.1 \pm 0.3$ & 0.16 & Pre-II ST & 0 & 1.0 \\
\hline Post-II ST & $0.4 \pm 0.5$ & & Post-II ST & $0.1 \pm 0.3$ & & Post-II ST & 0 & \\
\hline Pre-III ST & 0 & 0.32 & Pre-III ST & $-0.1 \pm 0.3$ & 0.32 & Pre-III ST & 0 & 1.0 \\
\hline Post-III ST & $0.2 \pm 0.4$ & & Post-III ST & 0 & & Post-III ST & 0 & \\
\hline Pre-aVR ST & 0 & 1.0 & Pre-aVR ST & 0 & 0.32 & Pre-aVR ST & 0 & 1.0 \\
\hline Post-aVR ST & 0 & & Post-aVR ST & $0.1 \pm 0.3$ & & Post-aVR ST & 0 & \\
\hline Pre-aVL ST & 0 & 1.0 & Pre-aVL ST & $0.1 \pm 0.3$ & 0.32 & Pre-aVL ST & 0 & 1.0 \\
\hline Post-aVL ST & 0 & & Post-aVL ST & 0 & & Post-aVL ST & 0 & \\
\hline Pre-aVF ST & $0.4 \pm 0.5$ & 1.0 & Pre-aVF ST & $-0.1 \pm 0.4$ & 0.08 & Pre-aVF ST & 0 & 1.0 \\
\hline Post-aVF ST & $0.4 \pm 0.5$ & & Post-aVF ST & $0.1 \pm 0.3$ & & Post-aVF ST & 0 & \\
\hline Pre-V1 ST & $0.2 \pm 0.4$ & 1.0 & Pre-V1 ST & $0.1 \pm 0.3$ & 1.0 & Pre-V1 ST & 0 & 1.0 \\
\hline Post-V1 ST & $0.2 \pm 0.4$ & & Post-V1 ST & $0.1 \pm 0.3$ & & Post-V1 ST & 0 & \\
\hline Pre-V2 ST & $0.3 \pm 0.7$ & 0.32 & Pre-V2 ST & 0 & $0.04^{*}$ & Pre-V2 ST & $0.2 \pm 0.4$ & 0.18 \\
\hline Post-V2 ST & $0.5 \pm 0.7$ & & Post-V2 ST & $0.3 \pm 0.5$ & & Post-V2 ST & $0.7 \pm 0.7$ & \\
\hline Pre-V3 ST & $0.5 \pm 0.7$ & 1.0 & Pre-V3 ST & $0.1 \pm 0.7$ & 0.32 & Pre-V3 ST & $0.2 \pm 0.4$ & 0.10 \\
\hline Post-V3 ST & $0.5 \pm 0.7$ & & Post-V3 ST & $0.4 \pm 0.6$ & & Post-V3 ST & $0.9 \pm 0.8$ & \\
\hline Pre-V4 ST & 0 & 0.16 & Pre-V4 ST & $-0.03 \pm 0.6$ & 0.17 & Pre-V4 ST & $0.2 \pm 0.4$ & 0.18 \\
\hline Post-V4 ST & $0.4 \pm 0.5$ & & Post-V4 ST & $0.2 \pm 0.5$ & & Post-V4 ST & $0.5 \pm 0.7$ & \\
\hline Pre-V5 ST & $0.4 \pm 0.5$ & 0.32 & Pre-V5 ST & $-0.1 \pm 0.5$ & 0.18 & Pre-V5 ST & 0 & 1.0 \\
\hline Post-V5 ST & $0.2 \pm 0.4$ & & Post-V5 ST & $0.1 \pm 0.4$ & & Post-V5 ST & 0 & \\
\hline Pre-V6 ST & $0.2 \pm 0.4$ & 0.32 & Pre-V6 ST & $-0.1 \pm 0.4$ & 0.16 & Pre-V6 ST & 0 & 1.0 \\
\hline Post-V6 ST & 0 & & Post-V6 ST & 0 & & Post-V6 ST & 0 & \\
\hline Pre-I R & $2.6 \pm 1.8$ & 0.28 & Pre-I R & $2.7 \pm 1.1$ & 0.18 & Pre-I R & $1.8 \pm 0.4$ & 0.26 \\
\hline Post-I R & $1.6 \pm 0.5$ & & Post-I R & $3.2 \pm 1.6$ & & Post-I R & $3.2 \pm 2.3$ & \\
\hline Pre-II R & $8.2 \pm 3.1$ & 0.83 & Pre-II R & $8.2 \pm 3.5$ & 0.18 & Pre-II R & $5.4 \pm 3.1$ & 0.26 \\
\hline Post-II R & $8 \pm 4.2$ & & Post-II R & $7 \pm 3.0$ & & Post-II R & $6 \pm 2.8$ & \\
\hline Pre-III $R$ & $5.8 \pm 4.0$ & 0.79 & Pre-III R & $6.1 \pm 3.2$ & 0.06 & Pre-III R & $5.0 \pm 2.9$ & 0.58 \\
\hline Post-III R & $5.8 \pm 3.0$ & & Post-III R & $4.6 \pm 2.7$ & & Post-III R & $4.4 \pm 2.5$ & \\
\hline Pre-aVR R & $2.4 \pm 1.9$ & 0.66 & Pre-aVR R & $2.6 \pm 2.1$ & 0.38 & Pre-aVR R & $1.2 \pm 1.1$ & 0.16 \\
\hline Post-aVR R & $2.2 \pm 1.8$ & & Post-aVR R & $2.4 \pm 2.3$ & & Post-aVR R & $1.6 \pm 1.1$ & \\
\hline Pre-aVL R & $1.2 \pm 0.8$ & 0.1 & Pre-aVL R & $1.3 \pm 1.2$ & 0.83 & Pre-aVL R & 0 & 0.28 \\
\hline Post-aVL R & $0.4 \pm 0.9$ & & Post-aVL R & $1.4 \pm 0.9$ & & Post-aVL R & $1.0 \pm 1.2$ & \\
\hline Pre-aVF R & $6.4 \pm 3.4$ & 1.0 & Pre-aVF R & $6.6 \pm 3.5$ & 0.08 & Pre-aVF R & $4.6 \pm 2.6$ & 1.0 \\
\hline Post-aVF R & $6.4 \pm 3.4$ & & Post-aVF R & $5.4 \pm 2.6$ & & Post-aVF R & $4.6 \pm 2.6$ & \\
\hline Pre-V1 R & $2.2 \pm 1.1$ & 0.41 & Pre-V1 R & $2.5 \pm 1.5$ & 0.32 & Pre-V1 R & $4.6 \pm 4.8$ & 0.29 \\
\hline Post-V1 R & $1.8 \pm 1.3$ & & Post-V1 R & $2.0 \pm 1.4$ & & Post-V1 R & $2.0 \pm 1.0$ & \\
\hline Pre-V2 R & $3.6 \pm 1.5$ & 0.41 & Pre-V2 R & $3.1 \pm 1.7$ & 0.96 & Pre-V2 R & $4.8 \pm 4.7$ & 0.74 \\
\hline Post-V2 R & $3.0 \pm 1.4$ & & Post-V2 R & $3.2 \pm 2.4$ & & Post-V2 R & $4.2 \pm 3.6$ & \\
\hline Pre-V3 R & $6.2 \pm 4.5$ & 0.46 & Pre-V3 R & $4.7 \pm 2.7$ & 0.61 & Pre-V3 R & $5.4 \pm 4.9$ & 0.71 \\
\hline Post-V3 R & $4.4 \pm 1.7$ & & Post-V3 R & $5.3 \pm 3.6$ & & Post-V3 R & $5.0 \pm 3.7$ & \\
\hline Pre-V4 R & $10 \pm 9.8$ & 0.49 & Pre-V4 R & $7.1 \pm 5.1$ & 0.51 & Pre-V4 R & $5.4 \pm 3.8$ & 0.59 \\
\hline Post-V4 R & $6.6 \pm 2.3$ & & Post-V4 R & $8.5 \pm 5.4$ & & Post-V4 R & $6.6 \pm 4.9$ & \\
\hline Pre-V5 R & $10.2 \pm 8.6$ & 0.68 & Pre-V5 R & $7.8 \pm 5.8$ & 0.59 & Pre-V5 R & $3.6 \pm 1.3$ & 0.07 \\
\hline Post-V5 R & $7.0 \pm 3.3$ & & Post-V5 R & $8.1 \pm 5.7$ & & Post-V5 R & $8.0 \pm 5.5$ & \\
\hline Pre-V6 R & $7.6 \pm 5.4$ & 0.14 & Pre-V6 R & $7.1 \pm 4.8$ & 0.65 & Pre-V6 R & $3.2 \pm 1.3$ & 0.07 \\
\hline Post-V6 R & $5.6 \pm 3.1$ & & Post-V6 R & $6.0 \pm 4.0$ & & Post-V6 R & $7.8 \pm 5.5$ & \\
\hline
\end{tabular}

Continuous variables are presented as mean \pm standard deviation.

ECG, electrocardiographic; Pre, before closed thoracostomy state; Post, after closed thoracostomy state.

${ }^{*} \mathrm{P}<0.05$ is statistically significant. 
Table 4. Changes in ST segments after closed thoracostomy

\begin{tabular}{llcc}
\hline & & $\begin{array}{c}\text { ST abnormality before } \\
\text { thoracostomy }\end{array}$ & $\begin{array}{c}\text { ST normalized } \\
\text { after thoracostomy }\end{array}$ \\
\hline Total $(n=45)$ & $\operatorname{RP}(n=20)$ & $8(40)$ & $2(25)$ \\
& $\operatorname{LP}(n=25)$ & $6(24)$ & $2(33)$ \\
$\operatorname{RP}(n=20)$ & $<30 \%(n=3)$ & 1 & 0 \\
& $30 \%-80 \%(n=9)$ & 3 & 1 \\
& $>80 \%(n=8)$ & 4 & 1 \\
$\operatorname{LP}(n=25)$ & $<30 \%(n=5)$ & 1 & 0 \\
& $30 \%-80 \%(n=15)$ & 4 & 2 \\
& $>80 \%(n=5)$ & 1 & 0 \\
\hline
\end{tabular}

Values are presented as number (\%) or number.

$\mathrm{RP}$, right-sided pneumothorax; $L P$, left-sided pneumothorax.
Table 5. Changes in axis after closed thoracostomy

\begin{tabular}{lcc}
\hline & \multicolumn{2}{c}{ Normalized axis after thoracostomy } \\
\hline Total axis deviation $(n=15)$ & \multicolumn{2}{c}{$8(53)$} \\
\hline & $\begin{array}{c}\text { Clockwise changes } \\
\text { in axis after } \\
\text { thoracostomy }\end{array}$ & $\begin{array}{c}\text { Counterclockwise } \\
\text { changes in axis } \\
\text { after thoracostomy }\end{array}$ \\
\hline RP $(n=20)$ & $9(45)$ & $11(55)$ \\
LP $(n=25)$ & $10(40)$ & $14(56)$ \\
\hline
\end{tabular}

Values are presented as number (\%).

$\mathrm{RP}$, right-sided pneumothorax; LP, left-sided pneumothorax.

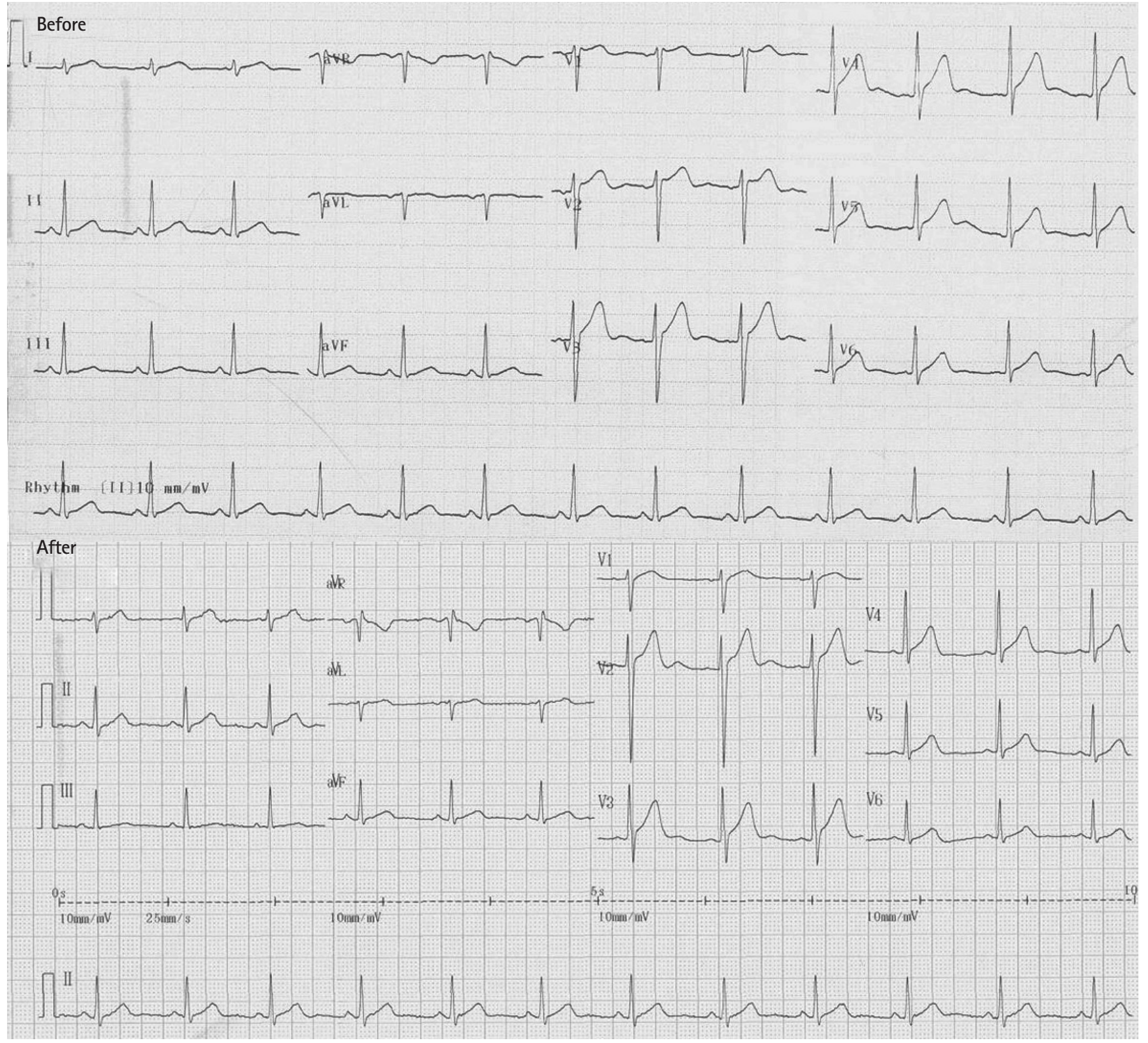

Fig. 1. A case with right-sided 72\% pneumothorax. The axis was changed from a normal axis to a right axis deviation after closed thoracostomy. 


\section{Changes in axis after closed thoracostomy}

The number of cases with axis deviation was 15. Among these cases, 8 had a normalized axis after closed thoracostomy (Table 5). Among the right-sided pneumothorax cases, the numbers of cases with clockwise and counterclockwise changes in axis were 9 and 11, respectively. Among the left-sided pneumothorax cases, the numbers of cases with clockwise and counterclockwise changes in axis were 10 and 14, respectively.
Moreover, in 1 case of a 72\% volume of right-sided pneumothorax, the axis was changed from a normal axis to a right axis deviation after closed thoracostomy (Fig. 1). However, in 1 case of left-sided pneumothorax with a 78\% volume, the axis was not changed after closed thoracostomy. In this case, left axis deviation $\left(-70^{\circ}\right)$ was present both before and after closed thoracostomy (Fig. 2).

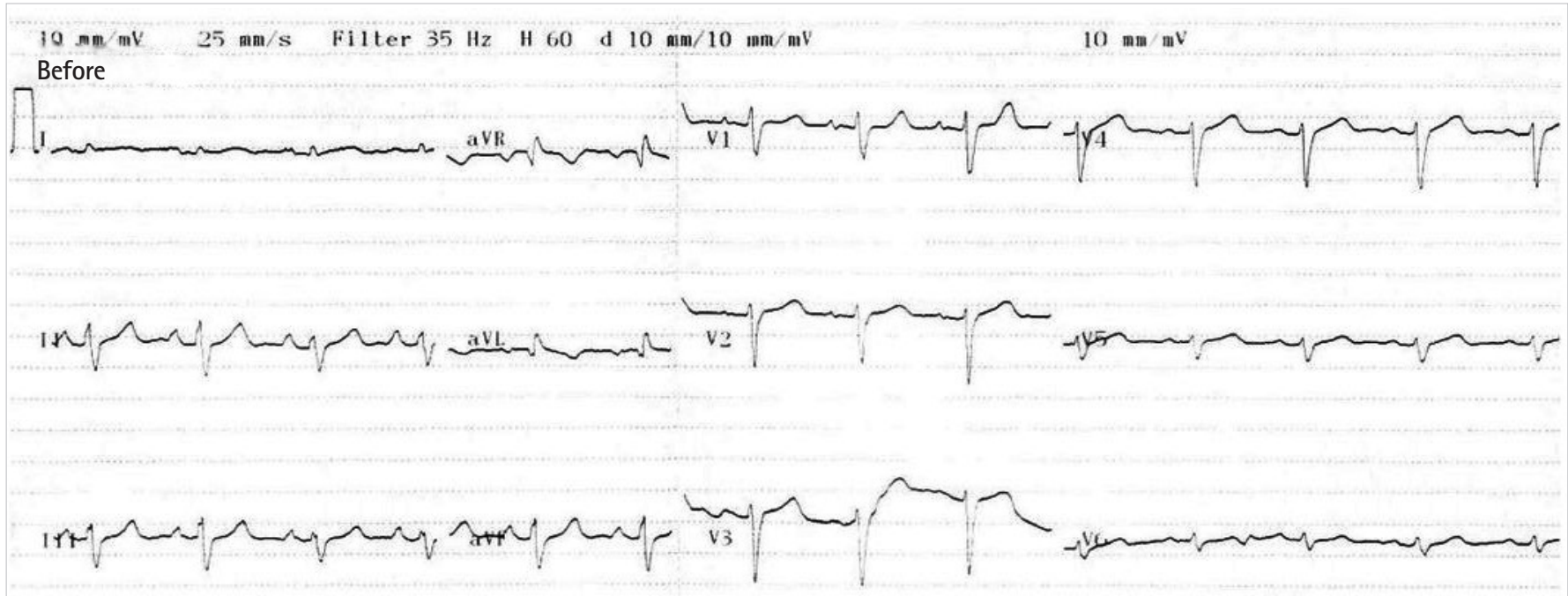

Rivition

$10 \mathrm{~mm} / \mathrm{mV} \quad 25 \mathrm{~mm} / \mathrm{s}$ Filter $35 \mathrm{~Hz}$ H 60 d $10 \mathrm{~mm} / 10 \mathrm{~mm} / \mathrm{mV}$

$10 \mathrm{~mm} / \mathrm{mV}$

Rifter

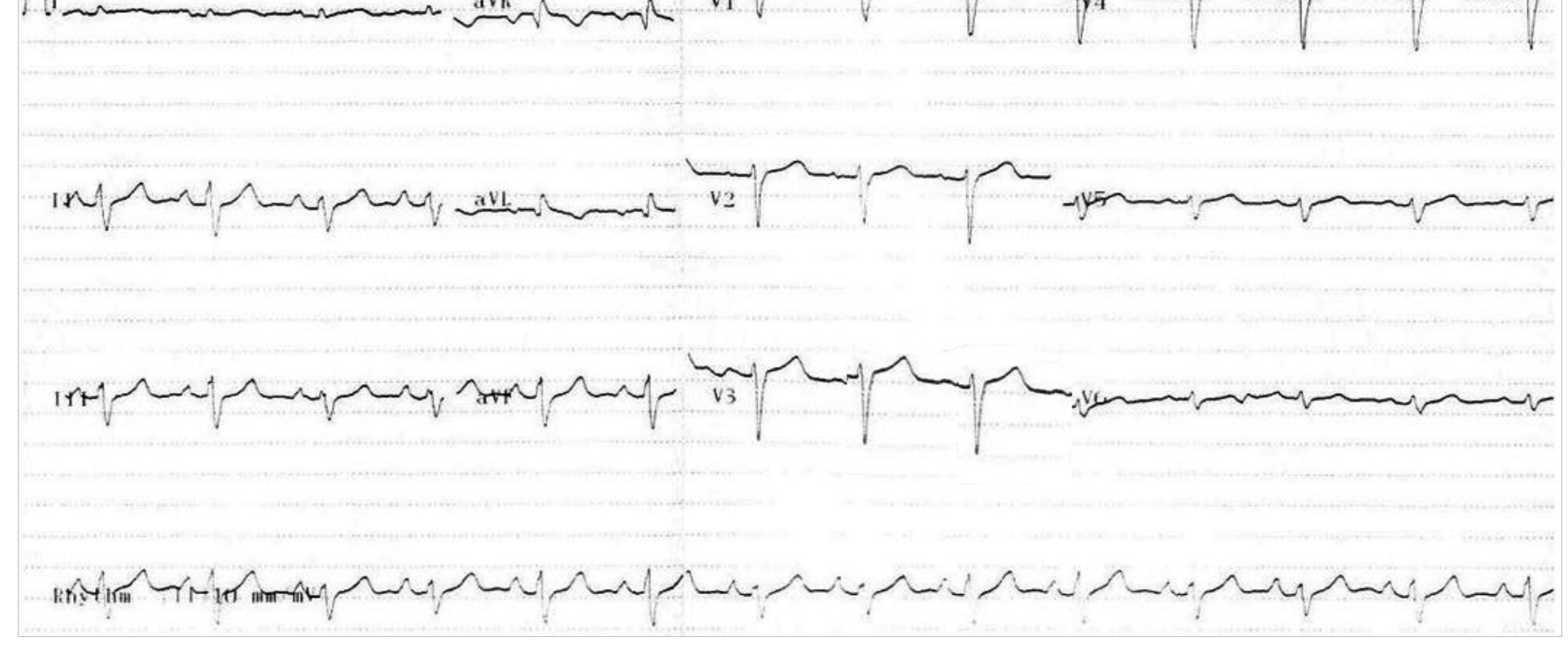

Fig. 2. A case with left-sided 78\% pneumothorax. The axis was not changed after closed thoracostomy. 
Table 6. Patients with abnormal ST segment changes

\begin{tabular}{ccccclc}
\hline Patient no. & Age (yr) & Sex & Side & Volume (\%) & \multicolumn{1}{c}{$\begin{array}{c}\text { Initial ST segment changes } \\
\text { ST segment normalized after } \\
\text { thoracostomy }\end{array}$} \\
\hline 1 & 18 & M & Right & 35 & ST elevation in V2, V3, V4, V5 & Yes \\
2 & 18 & M & Right & 83 & ST elevation in V4, V5, V6 & Yes \\
3 & 16 & M & Left & 24 & ST elevation in aVF, V1, V2, V3, V5, V6 & No \\
4 & 18 & M & Left & 50 & ST elevation in V3, V4 & No \\
5 & 18 & M & Right & 85 & ST elevation in I, aVL, V2, V3, V4, V5, V6 & No \\
6 & 18 & M & Left & 34 & ST elevation in V3, V4, V5 & No \\
7 & 20 & M & Right & 33 & ST elevation in V2, V3, V4 & No \\
8 & 45 & M & Left & 81 & ST elevation in V2, V3, V4 & No \\
9 & 18 & M & Right & 72 & ST elevation in V1, V2, V3, V4, V5 & No \\
10 & 23 & M & Right & 85 & ST elevation in II, III, aVF, V3, V4, V5 & Yes \\
11 & 67 & M & Left & 36 & ST depression in V3, V4, V5, V6 & No \\
12 & 30 & M & Right & 82 & ST elevation in V2, V3 & No \\
13 & 18 & M & Right & 23 & ST elevation in V3, V4, V5 & Yes \\
14 & 67 & M & Left & 48 & ST depression in II, III, aVF, V3, V4, V5, V6 & \\
\hline
\end{tabular}

\section{Patients with abnormal ST segment changes}

The number of cases with abnormal ST segment changes was 14 . Table 6 shows that abnormal ST segment changes occurred in pneumothorax cases of various sides and volumes. These findings were resolved by using closed thoracostomy in only 4 cases (Table 6).

\section{DISCUSSION}

Acute chest pain and dyspnea are common symptoms in patients who visit the emergency department. These symptoms are the manifestations of various conditions such as myocardial infarction, pulmonary embolism, pneumonia, pleural effusion, and pneumothorax. For these patients, electrocardiography is one of the most important diagnostic methods used in the differential diagnosis of such diseases. In most cases, ECG abnormalities are related to cardiac disease. However, many non-cardiac conditions may affect ECG findings such as electrolyte imbalance, thyroid diseases, and drug use. In addition, pneumothorax is not related to cardiac disease but may affect ECG findings, although it is a rare condition among non-cardiac conditions.

The first reports on pneumothorax-related ECG changes were provided in certain previous studies. ${ }^{1,9}$ Based on these studies, tuberculosis specialists were the first to be concerned with pneumothorax-related ECG changes. Specifically the use of artificial pneumothorax for the treatment of tuberculosis provided an opportunity to compare electrocardiograms obtained before and after induction of pneumothorax. However, in one study, the obtained ECG findings were less informative, as the researchers used few leads. In another study, the researchers reported differences in ECG abnormalities between patients with artificial and those with spontaneous pneumothorax. However, for the above-men- tioned reasons, the study was limited. ${ }^{9}$

Generally, reports have included individual cases of ECG changes mimicking pulmonary embolism, ${ }_{1}^{2}$ myocardial infarction, ${ }_{1}^{3,4}$ ischemia, ${ }_{1}^{10}$ or anecdotal ECG changes such as breathing-dependent QRS amplitude or PR length. ${ }^{8,11}$

In a previous study, researchers reported that an abnormal axis is more common in patients with left-sided pneumothorax than in those with right-sided pneumothorax, while changes in ORS morphology (most commonly right bundle branch block) and T waves (inversion) appeared more often in patients with right-sided pneumothorax. The latter finding was only observed in patients with massive pneumothorax. ${ }^{6}$ In addition, the researchers reported that the changes in QRS amplitude in the leads in pneumothorax patients were most likely secondary to alterations in the distance between the heart and the chest wall, and to changes in electric impulse conduction pathways. ${ }^{6}$

However, in our results, we found no definite significant trends except for several findings according to the side and volume of the pneumothorax. In this study, in the right-sided pneumothorax cases with a pneumothorax volume of $>80 \%$, a OTc interval change was the most significant finding. This means that massive pneumothorax affected the impedance between the chest wall and the heart. However, in the left-sided pneumothorax cases with the same volume range, a OTc interval change was not a statistically significant finding $(P=0.14)$. This finding indicates that even when the pneumothorax is massive, because of the shorter distance between the chest wall and the heart on the left side of the chest than on the right side, the effect on the impedance was smaller on the left side than on the right side.

In contrast to the results of previous studies, ${ }^{6}$ the results of the present study indicated no statistically significant changes in cas- 
es of left-sided pneumothorax with a pneumothorax volume of $>80 \%$. If anything, we found statistically significant changes in cases with a pneumothorax volume between $30 \%$ and $80 \%$, and not in massive pneumothorax.

Intrapleural free air may restrict cardiac functional movement and cause compression of coronary vessels. Increased intrathoracic pressure could decrease venous return and stroke volume, ultimately inducing tachycardia. In turn, tachycardia aggravates oxygen demand, shortens diastolic perfusion, and leads to ischemia of coronary vessels. ${ }^{5}$ Certain investigators reported that ST segment elevation or depression could be induced by tension pneumothorax. ${ }^{12}$ In particular, they reported that patients who presented with pneumothorax were initially diagnosed as having acute coronary syndrome. ${ }^{12}$ Several patients were treated with a thrombolytic agent or emergency coronary angiography before the exact diagnosis of pneumothorax could be made. ${ }^{12}$ Furthermore, a case of myocardial infarction in a patient with tension pneumothorax was reported. ${ }^{13,14}$ All studies indicated that physicians should be aware that reversible ECG changes and specifically ST segment depression and elevation are not uncommon in patients with spontaneous pneumothorax. ${ }^{5}$ In the present study, ST segment changes in the precordial lead V2 were statistically significant after closed thoracostomy only in cases of a left-sided pneumothorax volume between $30 \%$ and $80 \%$. However, these changes in only one precordial lead were not clinically significant. Generally, the number of cases of ST segment abnormality in rightsided pneumothorax was 8 (40\%). Among these cases, only 2 cases normalized after closed thoracostomy. By contrast, the number of cases of ST segment abnormality in left-sided pneumothorax was 6 (24\%). Among these cases, only 2 normalized after closed thoracostomy. Although we investigated these phenomena according to volume ranges, we could not find any trends.

If abnormal ST segment changes are due to pneumothorax, these findings must be resolved after resolution of pneumothorax by using closed thoracostomy. ${ }^{2,15,16}$ However, in the present study, ST segment abnormalities resolved in only 4 cases. These results show that it is wrong to conclude that abnormal ST segment findings are better represented when the pneumothorax is of a greater volume. Of course, however, we do not know why ST segment changes occurred in cases of low-volume pneumothorax or why these findings were not resolved after resolution of pneumothorax by using closed thoracostomy.

Among the results of this study, those for the axis would be as expected. In one case, the axis in a patient with a right-sided pneumothorax with $72 \%$ volume was changed to a right-axis deviation after closed thoracostomy. Moreover, changes in the axis in several cases were consistent with previous studies. ${ }^{5,6,9,11,15}$ How- ever, in contrast to previous studies, the present study found that for right-sided pneumothorax, the axis of 11 cases was changed to the counterclockwise direction after closed thoracostomy, and for left-sided pneumothorax, the axis of 10 cases was changed to the clockwise direction after closed thoracostomy. However, in one case, the axis of a left-sided pneumothorax with $78 \%$ volume was not changed after closed thoracostomy. The change in axis indicates that the heart was pushed by the massive pneumothorax, and consequently, an actual change in the axis occurred. Otherwise, the axis might have appeared exaggerated because of a change in impedance. In addition, the mechanism of the one case in which the axis of a left-sided pneumothorax with 78\% volume was not changed after closed thoracostomy could not be fully understood because of the retrospective nature of the data analysis in this study.

This study has several limitations. First, this study was conducted by using a retrospective data analysis. Generally, retrospective data may be clear and may be satisfactory for our purposes. Therefore, an analysis of these data may be appropriate. However, because we excluded data that did not satisfy our inclusion criteria, these data may not be broadly credible. Second, the total number of enrolled patients was 45 , which is higher than those in any previous studies. However, in each volume range, the sample size was very small. Furthermore, these data were collected at a single urban tertiary medical center. These conditions might have affected the analysis of the results. Third, no ECG data were available from before the onset of the disease. For this reason, a more precise analysis of the results in this study was not possible. However, because we investigated the changes in ECG findings after closed thoracostomy in patients with spontaneous pneumothorax, we might have somewhat overcome this limitation. Fourth, we did not perform a statistical analysis by using the delta value of the axis before and after closed thoracostomy. If we were to conduct this statistical analysis, we would obtain a relationship between the side of the pneumothorax and the axis, and between the volume of the pneumothorax and the axis.

In conclusion, similar to the results of previous studies, changes in several pneumothorax-related ECG findings were found on both sides, as expected. However, in this study, the trend found in previous studies was not prominent. In addition, previous reports about ST segment changes due to the volume of the pneumothorax were found to be wrong. Therefore, for differential diagnosis, careful physical examination in the emergency department should be performed for patients with chest pain with or without dyspnea. In addition, radiography and electrocardiography must first be performed to avoid making the wrong decision and acting based on ECG findings alone. 


\section{CONFLICT OF INTEREST}

No potential conflict of interest relevant to this article was reported.

\section{REFERENCES}

1. Armen RN, Frank TV. Electrocardiographic patterns in pneumothorax. Dis Chest 1949;15:709-19.

2. Goddard R, Scofield RH. Right pneumothorax with the S103T3 electrocardiogram pattern usually associated with pulmonary embolus. Am J Emerg Med 1997;15:310-2.

3. Diamond JR, Estes NM. ECG changes associated with iatrogenic left pneumothorax simulating anterior myocardial infarction. Am Heart J 1982;103:303-5.

4. Raev D. A case of spontaneous left-sided pneumothorax with ECG changes resembling acute myocardial infarction. Int J Cardiol 1996;56:197-9.

5. Senthilkumaran $S$, Meenakshisundaram R, Michaels AD, Thirumalaikolundusubramanian $\mathrm{P}$. Electrocardiographic changes in spontaneous pneumothorax. Int J Cardiol 2011;153:78-80.

6. Krenke R, Nasilowski J, Przybylowski T, Chazan R. Electrocardiographic changes in patients with spontaneous pneumothorax. J Physiol Pharmacol 2008;59 Suppl 6:361-73.

7. Slay RD, Slay LE, Luehrs JG. Transient ST elevation associated with tension pneumothorax. JACEP 1979;8:16-8.

8. Kozelj $M$, Rakovec $P$, Sok M. Unusual ECG variations in leftsided pneumothorax. J Electrocardiol 1997;30:109-11.

9. Master AM. The electrocardiographic changes in pneumothorax in which the heart has been rotated: the similarity of some of these changes to those indicating myocardial involvement. Am Heart J 1928;3:472-83.

10. Ruo W, Rupani G. Left tension pneumothorax mimicking myocardial ischemia after percutaneous central venous cannulation. Anesthesiology 1992;76:306-8.

11. Strizik B, Forman R. New ECG changes associated with a tension pneumothorax: a case report. Chest 1999;115:1742-4.

12. Sanders A, Froude A, Probst F. Do we intervene inappropriately for ST elevation? Emerg Med J 2006;23:e10.

13. Janssens $U$, Koch $K C$, Graf J, Hanrath P. Severe transmyocardial ischemia in a patient with tension pneumothorax. Crit Care Med 2000;28:1638-41.

14. Chan WH, Lin CS, Yang SP, Cheng SM. ECG changes with elevated troponin I in a patient with tension pneumothorax. South Med J 2009;102:969-71.

15. Kamimura $M$, Kudo $K$, Kubota $T$, School JM. ECG changes in tension pneumothorax: a hypothesis. Chest 2000;117:1527.

16. Walston A, Brewer DL, Kitchens CS, Krook JE. The electrocardiographic manifestations of spontaneous left pneumothorax. Ann Intern Med 1974;80:375-9. 